\title{
Genome-wide association mapping unravels the genetic control of seed germination and vigor in Brassica napus
}

\author{
Sarah V. Hatzig ${ }^{1}$, Matthias Frisch ${ }^{2}$, Frank Breuer ${ }^{3}$, Nathalie Nesi ${ }^{4}$, Sylvie Ducournau ${ }^{5}$, \\ Marie-Helene Wagner ${ }^{5}$, Gunhild Leckband ${ }^{6}$, Amine Abbadi ${ }^{6}$ and Rod J. Snowdon ${ }^{1 *}$ \\ ${ }^{1}$ Department of Plant Breeding, Justus Liebig University, Giessen, Germany, ${ }^{2}$ Department of Biometry and Population \\ Genetics, Justus Liebig University, Giessen, Germany, ${ }^{3}$ KWS SAAT AG, Einbeck, Germany, ${ }^{4}$ Institute for Genetics, \\ Environment and Plant Protection, INRA, Le Rheu, France, ${ }^{5}$ Groupe d'Etude et de contrôle des Variétés Et des Semences, \\ Beaucouzé, France, ${ }^{6} \mathrm{NPZ}$ Innovation GmbH, Holtsee, Germany
}

OPEN ACCESS

Edited by:

Rajeev K. Varshney, International Crops Research Institute for the Semi-Arid Tropics, India

Reviewed by:

Dongying Gao,

University of Gerogia, USA

Zhe Liang,

National University of Singapore,

Singapore

*Correspondence:

Rod J. Snowdon,

Department of Plant Breeding, Justus Liebig University, IFZ Research Centre

for Biosystems, Land Use and Nutrition, Heinrich-Buff-Ring 26-32,

35392 Giessen, Germany

rod.snowdon@agrar.uni-giessen.de

Specialty section:

This article was submitted to Plant Genetics and Genomics, a section of the journal Frontiers in Plant Science

Received: 20 February 2015 Accepted: 20 March 2015 Published: 09 April 2015

Citation: Hatzig SV, Frisch M, Breuer F, Nesi N, Ducournau S, Wagner M-H, Leckband G, Abbadi A and Snowdon RJ (2015) Genome-wide association mapping unravels the genetic control of seed germination and vigor in Brassica napus. Front. Plant Sci. 6:221. doi: 10.3389/fpls.2015.00221
Rapid and uniform seed germination is a crucial prerequisite for crop establishment and high yield levels in crop production. A disclosure of genetic factors contributing to adequate seed vigor would help to further increase yield potential and stability. Here we carried out a genome-wide association study in order to define genomic regions influencing seed germination and early seedling growth in oilseed rape (Brassica napus L.). A population of 248 genetically diverse winter-type B. napus accessions was genotyped with the Brassica 60k SNP Illumina genotyping array. Automated high-throughput in vitro phenotyping provided extensive data for multiple traits related to germination and early vigor, such as germination speed, absolute germination rate and radicle elongation. The data obtained indicate that seed germination and radicle growth are strongly environmentally dependent, but could nevertheless be substantially improved by genomic-based breeding. Conditions during seed production and storage were shown to have a profound effect on seed vigor, and a variable manifestation of seed dormancy appears to contribute to differences in germination performance in B. napus. Several promising positional and functional candidate genes could be identified within the genomic regions associated with germination speed, absolute germination rate, radicle growth and thousand seed weight. These include B. napus orthologs of the Arabidopsis thaliana genes SNOWY COTYLEDON 1 (SCO1), ARABIDOPSIS TWO-COMPONENT RESPONSE REGULATOR (ARR4), and ARGINYL-t-RNA PROTEIN TRANSFERASE 1 (ATE1), which have been shown previously to play a role in seed germination and seedling growth in A. thaliana.

Keywords: seedling, emergence, high-throughput phenotyping, GWAS, rapeseed, canola

\section{Introduction}

Selection bottlenecks have a large impact on the diversity available for breeders to sustain selection gains for important traits, particularly in crop species like modern oilseed rape (Brassica napus L.) with relatively small gene pools. One approach to overcome this problem is introgression of 
untapped germplasm in order to broaden the genetic basis of breeding materials (Friedt and Snowdon, 2010). A main focus in genetics and breeding of oilseed rape has been on analysis and improvement of general breeding targets, such as abiotic and biotic stress resistances (e.g., Obermeier et al., 2013; Hatzig et al., 2014) or flowering time optimization (Schiessl et al., 2014). Enhancement of such traits can contribute directly to an increase and stabilization of yield levels. On the other hand, less attention has been given to elucidation of the genetic control of seed germination and seedling vigor, although these are fundamental processes shaping field emergence (Matthews and Khajeh Hosseini, 2006; Wagner et al., 2011; Matthews et al., 2012) and yield performance (Larsen et al., 1998).

Germination is a major component of seed vigor, which is defined as the sum of those properties of the seed that determine the potential level of activity and performance of seed lots with acceptable germination under a wide range of environments (Perry, 1978). Although seed germination is influenced by both genetic as well as environmental factors, it is still an open question whether seeds performing well under optimal conditions also have advantages under stress conditions. However, different studies dealing with drought (Bettey et al., 2000), salt or cold stress (Foolad et al., 1999) strengthen such correlations.

Germination per se commences with the uptake of water by the quiescent dry seed, interruption of dormancy and the subsequent elongation of the embryonic axis (Bewley, 1997). Water uptake during germination is a triphasic process while per definition germination is completed upon entry into the third phase (Bewley, 1997). Initially, water uptake is driven by physical swelling of the seed when hydrophilic molecular groups form hydration shells. There is evidence that environmental influences already affect germination during this initial phase, in which water uptake takes place independently from metabolism. Factors like temperature or the presence of specific solutes are able to retard or accelerate seed swelling (Leopold, 1983). Subsequent to physical swelling, metabolic processes are initiated and germination becomes irreversible by water deprivation. This second phase is also described as the plateau phase (Bewley, 1997), as increases in fresh weight due to physical swelling are already completed, while active growth takes place due to cell differentiation and elongation after the initiation of underlying biochemical processes. Per definition germination stops upon entry into the

\footnotetext{
Abbreviations: $\mathrm{ABA}, \mathrm{Abscisic}$ acid; ARR4, A. thaliana gene two-component response regulator ARR4 (AT1G10470.1); ATE1, A. thaliana gene Arginine-tRNA Protein Transferase 1 (AT5G05700.1); Bna.ARR4, B. napus ortholog of A. thaliana two-component response regulator ARR4 (AT1G10470.1); Bna.ATE1, B. napus ortholog of A. thaliana gene ATE1, encoding for Arginine-tRNA Protein Transferase 1 (AT5G05700.1); Bna.SCO1, B. napus ortholog of A. thaliana gene snowy cotyledon 1 (AT1G62750.1); DMSO, Dimethyl sulfoxide; ES, Elongation speed of the radicle $(\mathrm{mm} / \mathrm{h}) ; \mathrm{FG}$, First germination $(\mathrm{h}) ; \mathrm{F}_{\mathrm{ST}}$, Fixation index; GO, Gene onthology; GR36, Germination rate within 36h (\%); GR72, Germination rate within $72 \mathrm{~h}(\%)$; GWAS, Genome-wide association study; IBD, Identity by descent; IS, Imbibition speed during first $4 \mathrm{~h}$ after initiation of imbibition $\left(\mathrm{mm}^{3} / \mathrm{h}\right)$; LD, Linkage disequilibrium; MGT, Mean germination time (h); PC, Principal component; PCA, Principal component analysis; SCO1, A. thaliana gene snowy cotyledon 1 (AT1G62750.1); SL2011, Seed lot produced in 2011 in LeRheu, France; SL2012, Seed lot produced in 2012 in Asendorf, Germany; SNP, Single nucleotide polymorphism; T50, Time necessary to reach $50 \%$ of germination (h); TSW, Thousand seed weight (g); VI, Volume increase within first $8 \mathrm{~h}(\%)$.
}

third phase, in which growth becomes visible and the primary root breaks through the testa (Bewley, 1997).

For breeding of vigorous plant cultivars with fast and uniform field emergence, it is crucial to understand the genetic factors that contribute to an adequate germination performance and seedling growth. As seed germination is a very complex trait controlled at transcriptional, translational and metabolic level (Rajjou et al., 2012), it is difficult to identify the contributing genetic factors by conventional genetic or physiological analyses. A preferential method for identification of genomic regions associated with complex quantitative traits is genome-wide association mapping, in which marker-trait associations are calculated across a broad set of diverse germplasm in order to define chromosome regions harboring promising genes.

The high resolution of genome-wide association studies, arising from the incorporation of numerous ancient meioses in a set of unrelated plant individuals, has become a popular method for disclosure of genomic polymorphisms affecting quantitative traits. However, a common drawback of GWAS is that population structure can shift allele frequencies within a diversity panel. This can lead to the detection of false-positive marker-trait associations (Korte and Farlow, 2013) or a non-detection of rare and/or region-specific alleles (Nordborg and Weigel, 2008). In such a case statistical methods have to be applied which correct for population stratification.

In this study we used genome-wide association studies for the investigation of quantitative trait loci (QTL) linked to germination performance and early vigor in winter oilseed rape (Brassica napus L.). An automated high throughput phenotyping platform (Demilly et al., 2014) was used to assay diverse traits, related to the three different phases of seed germination and post-germination radicle growth, in a large set of diverse winter rapeseed lines genotyped with genome-wide single-nucleotide polymorphism (SNP) markers. Genome-wide association analyses using these data sets enabled identification of highly promising candidate genes and markers for breeding towards improved germination in rapeseed. Phenotyping of seed lots produced in different environments helped to evaluate the effects of germination-independent factors, such as seed ripening and storage, on germination performance.

\section{Materials and Methods}

\section{Seed Material and Phenotyping}

A total of 248 genetically diverse, winter-type B. napus inbred lines were used in this study, including 216 winter oilseed rape (WOSR), 20 winter fodder (WF) and 10 exotic lines (Table S1). Seeds of all inbred lines were produced by controlled selfpollination in two distinct environments during the growing seasons of 2010/2011 (SL 2011) in Le Rheu, France and 2011/2012 (SL 2012) in Asendorf, Germany. The genotype panel comprises lines derived from modern oilseed rape cultivars with low seed erucic acid and glucosinolate content, old rapeseed varieties with high seed erucic acid and high glucosinolate content $(++$ quality), fodder rapes, kale vegetable forms, and resynthesized $B$. napus derived from interspecific hybridizations between its two diploid progenitors, Brassica rapa and Brassica oleracea. 
Monitoring of seed imbibition, germination, and early radicle growth was conducted under in vitro conditions using the automated phenotyping platform of the variety control office of the French national seed testing agency (Station Nationale d'Essais de Semences, Groupe d'Etude et de contrôle des Variétés et des Semences-GEVES, Angers, France). The phenotyping platform is described in detail by Ducournau et al. $(2004,2005)$ and Wagner et al. (2011). Image acquisition, image analysis and data analysis methods are described in detail by Demilly et al. (2014). The following parameters were determined: Volume increase within first $8 \mathrm{~h}$ (VI; in \%), imbibition speed during first $4 \mathrm{~h}$ after initiation of imbibition (IS; in $\mathrm{mm}^{3} / \mathrm{h}$ ), total germination rate within $72 \mathrm{~h}$ after initiation of imbibition (GR72; in \%), first germination time (FG; in h), mean germination time (MGT; in h), radicle elongation speed (ES; in $\mathrm{mm} / \mathrm{h}$ ), time to reach $50 \%$ of germination (T50; in $\mathrm{h}$ ) and germination rate within $36 \mathrm{~h}$ after initiation of imbibition (GR36; in \%). Additionally, thousand seed weight (TSW; in g) was measured before germination monitoring. Correlations were calculated applying the Pearson's product-moment correlation.

\section{Pre-Processing of Marker Data}

Genomic DNA was extracted from young leaf materials for all genotypes of the diversity panel $(n=248)$. Genotyping was performed with the Brassica $60 \mathrm{k}$ Illumina ${ }^{\circledR}$ Infinium consortium SNP array (Edwards et al., 2013), according to standard procedures of the manufacturer and using the same cluster file for SNP calling. For physical localization of SNP markers, flanking sequences were blasted onto the Brassica napus Darmor-bzh reference genome sequence assembly (version 4.1), recently published by Chalhoub et al. (2014). SNPs were blasted using the following criteria: minimum overlap of $50 \mathrm{bp}$ length, minimum identity of $95 \%$, no sequence gaps. SNPs which failed across the entire genotype collection, as well as all locus non-specific SNPs, for which more than 1 BLAST hit on the $B$. napus sequence was found, were excluded from further analyses. As heterozygous SNPs cannot be distinguished from multi-locus hemi-SNPs or false calls, heterozygous calls were treated as missing values. For visualization of population structure and calculation of genomewide associations, SNP markers with more than $20 \%$ missing calls across the panel were excluded. Furthermore, all individuals which had more than $20 \%$ missing calls across the genotype data were excluded. In order to incorporate rare alleles with potential effects on germination performance, only markers with a minor allele frequency $\leq 0.025$ were excluded from analysis. After preprocessing, 218 individuals and 22,169 SNP markers remained for further analyses.

\section{Population Structure Analysis}

Visualization of genetic relatedness was performed using the statistical software $\mathrm{R}$ studio version 0.98 .501 and the integrated R package SelectionTools (http://www.uni-giessen.de/ population-genetics/downloads). Genetic distances were calculated applying the euclidean modified Rodger's distance method according to Wright (1978). In order to visualize genetic relatedness among all genotypes, principal component analysis (PCA) was carried out regarding the first four components. Correction for population stratification was performed by a mixed-model approach including principal component covariates. According to Price et al. (2010) this is the method of choice for correction of population structure, family structure and cryptic relatedness. Genotypes were assigned to specific clusters by k-means clustering (MacQueen, 1967).

Optimal number of clusters was estimated by calculating the within-cluster sum of squares (WSS), varying cluster number $\mathrm{k}$ from 1 to 15 , and subsequently defined as 4 . Linkage disequilibrium (LD) was calculated for each chromosome individually, quoting coefficients of determination $\left(\mathrm{r}^{2}\right)$ for all locus pairs localized on the same chromosome. Inter-chromosomal genome-wide LD decay was calculated for trait-associated markers that exhibited unexpected patterns of local LD. Locally paired scatterplot smoothing in $\mathrm{R}$ was employed for graphical representation of LD curves with a span of 0.1 .

For characterization of genomic kinship, genome-wide allele identity by descent was computed for the whole diversity panel as well as separately for the four identified subpopulation clusters, using the $\mathrm{R}$ package GenABEL (Aulchenko et al., 2007). Additionally, to determine the proposition of genetic differentiation explained by differentiation among the subclusters, overall $\mathrm{F}_{\mathrm{ST}}$ values were calculated using the software Genepop version 4.2.2 (Rousset, 2008).

\section{Genome-Wide Association Analysis}

Narrow-sense heritability for each trait was calculated separately for each seed lot, again using the R package GenABEL. Genomewide associations were calculated with GenABEL. Adjustment for stratification was performed by mixed-model approximation combined with PC adjustment (Price et al., 2010; Svishcheva et al., 2012). For PC adjustment the first 4 components were taken into account.

False non-discovery rate was calculated with the $\mathrm{R}$ package fdrtool based on p-value statistics. Only associations exceeding the predetermined cutoff values were taken into account. For the integration of markers with weak associations to the phenotypic traits, all SNPs with a $-\log _{10}(p$-value $)>3$ were considered as significant. Marker-trait associations were regarded as reliable when they could be confirmed in a second seed lot with $-\log _{10}(p-$ value) $>2.5$. To define regions of interest for selection of potential candidate genes, local LD decay was first calculated within the flanking regions up to $1000 \mathrm{kbp}$ on either side of the associated markers. All genes anchored between the two markers next to an associated marker were taken into account for the disclosure of candidates. Furthermore, when flanking markers were in strong LD $\left(r^{2}>0.4\right)$ with an associated marker, regions were defined as LD blocks and the whole LD block was taken into account for candidate gene disclosure. Genes within LD blocks containing trait-associated markers were characterized with the tool Blast2GO (Conesa et al., 2005) using default settings, and candidates were selected based on their gene ontology (GO) terms. For differentiation between coding and non-coding regions of selected candidate genes the Genscan web server (http://genes. mit.edu/GENSCAN.html) was used. The plant pathway database MetNet (http://www.metnetonline.org) was used to determine 
common pathway memberships among the identified candidate genes.

Marker haplotypes were defined for each associated region and genotypes were bulked in regard to their associated-marker haplotypes. For T50 and ES boxplots were generated showing the phenotypic distribution of 4 and 6 classes, respectively, representing the cumulative phenotypes from haplotypes with favorable effects on the trait performance.

\section{Analysis of Chlorophyll Content}

For chlorophyll analyses, two groups of 10 extreme genotypes were selected with consistently high and low values, respectively, for ES (radicle elongation speed) in both seed lots. Seeds from SL2011 were used for chlorophyll content determination. Around 60 seeds of each genotype were germinated in Jacobsen vessels on circular filter papers soaked with $\mathrm{H}_{2} \mathrm{O}$ dest. Continuous water supply was ensured by wicks connecting the filter papers with $100 \mathrm{~mL} \mathrm{H}_{2} \mathrm{O}$ dest at the bottom of each vessel. Germination was carried out in a climate chamber with the following conditions: $20^{\circ} \mathrm{C}, 16 \mathrm{~h}$ light, $8 \mathrm{~h}$ dark. Chlorophyll extraction was performed after $5 \mathrm{~d}$. The cotyledons of eight seedlings per genotype were pooled and treated as one biological replicate. In total 3 biological replicates were sampled per genotype. Fresh weights of all samples were measured. Subsequently all samples were homogenized (Ultra-Thurrax T25, IKA, Staufen / Germany) in $15 \mathrm{~mL}$ Falcon tubes filled with $5 \mathrm{~mL}$ dimethyl sulfoxide (DMSO, >99.8\% GC). The samples were incubated at room temperature for $24 \mathrm{~h}$ in the dark. For chlorophyll quantification, $1 \mathrm{~mL}$ of the supernatant was collected and filled into a micro cuvette $(1.6 \mathrm{~mL})$ for spectrometric measurement. The absorbance of the sample solutions was measured after blank calibration (1 mL DMSO) with a SmartSpec Plus spectrophotometer (Bio-Rad Inc., Hercules, CA, USA) at $665 \mathrm{~nm}$ and $649 \mathrm{~nm}$ wavelength. Chlorophyll a and chlorophyll b concentrations were calculated according to the equations of Wellburn (1994). Significant differences were calculated with Student's $t$-test.

\section{Results}

\section{Phenotypic Distribution, Genetic Diversity and Linkage Disequilibrium}

Phenotypic values for all traits showed a similar mean trait performance and phenotypic variability in the two seed lots SL2011

TABLE 1 | Phenotypic variation for different traits associated with seed germination performance in two seeds lots of a Brassica napus diversity panel ( $\mathrm{n}=248$ ) produced in Le Rheu, France in 2011 (SL2011) and Asendorf, Germany in 2012 (SL2012), respectively: Minimum (Min), maximum (Max), standard deviation (SD) and correlation between seed lots (r).

\begin{tabular}{|c|c|c|c|c|c|c|c|c|c|c|}
\hline \multirow[t]{2}{*}{ Trait } & \multirow[t]{2}{*}{ Unit } & \multicolumn{4}{|c|}{ SL2011 } & \multicolumn{4}{|c|}{ SL2012 } & \multirow[b]{2}{*}{$r$} \\
\hline & & Min & Max & Mean & $S D$ & Min & Max & Mean & $S D$ & \\
\hline TSW & $g$ & 3.07 & 6.70 & 4.65 & 0.53 & 3.12 & 6.24 & 4.92 & 0.54 & $0.63^{\text {***}}$ \\
\hline $\mathrm{VI}$ & $\%$ & 8.12 & 40.37 & 22.33 & 5.08 & 7.91 & 46.43 & 24.14 & 6.57 & $0.44^{\star \star \star}$ \\
\hline IS & $\mathrm{mm}^{3} / \mathrm{h}$ & 0.02 & 0.35 & 0.18 & 0.05 & 0.00 & 0.40 & 0.17 & 0.07 & $0.24^{\star \star \star}$ \\
\hline$F G$ & $\mathrm{~h}$ & 12.00 & 30.00 & 21.85 & 3.81 & 12.00 & 28.00 & 17.43 & 3.35 & $0.26^{\star \star \star}$ \\
\hline MGT & $\mathrm{h}$ & 27.62 & 48.75 & 36.29 & 3.41 & 27.17 & 46.16 & 35.88 & 3.22 & $0.56^{\star \star \star}$ \\
\hline T50 & $\mathrm{h}$ & 26.14 & 62.00 & 35.14 & 3.88 & 27.08 & 45.75 & 35.20 & 3.22 & $0.57^{\star \star \star}$ \\
\hline GR36 & $\%$ & 7.00 & 93.33 & 55.89 & 18.62 & 10.00 & 87.00 & 54.78 & 15.73 & $0.58^{\star \star \star}$ \\
\hline GR72 & $\%$ & 44.00 & 100.00 & 96.96 & 5.53 & 75.00 & 100.00 & 94.68 & 4.00 & $0.44^{\star \star \star}$ \\
\hline ES & $\mathrm{mm} / \mathrm{h}$ & 0.51 & 1.24 & 0.81 & 0.12 & 0.61 & 1.23 & 0.88 & 0.12 & $0.57^{\star \star \star}$ \\
\hline
\end{tabular}

Abbreviations of traits are explained in Materials and Methods.

Significances were calculated at a level of ${ }^{\star \star *} p<0.001$.

TABLE 2 | Correlation coefficients (r) for different trait pairs associated with seed germination performance measured in two seed lots (SL2011 and SL2012) from an oilseed rape diversity panel $(n=248)$.

\begin{tabular}{|c|c|c|c|c|c|c|c|c|}
\hline & TSW & VI & IS & $\mathbf{F G}$ & MGT & T50 & GR36 & GR72 \\
\hline $\mathrm{Vl}$ & -0.04 & & & & & & & \\
\hline IS & $0.21^{\star \star *}$ & $0.74^{* * *}$ & & & & & & \\
\hline$F G$ & -0.08 & $-0.30^{\star \star \star}$ & $-0.10^{\star}$ & & & & & \\
\hline MGT & $0.10^{*}$ & $-0.41^{\star * *}$ & $-0.19^{\star * *}$ & $0.46^{* \star *}$ & & & & \\
\hline $\mathrm{T} 50$ & $0.15^{\star \star}$ & $-0.38^{\star \star \star}$ & $-0.18^{\star \star \star}$ & $0.36^{\star \star \star}$ & $0.94^{\star \star \star}$ & & & \\
\hline GR36 & $-0.15^{\star \star \star}$ & $0.39^{\star \star \star}$ & $0.19^{\star \star \star}$ & $-0.37^{\star \star \star}$ & $-0.95^{\star \star \star}$ & $-0.94^{\star \star \star}$ & & \\
\hline GR72 & -0.08 & $-0.14^{\star \star}$ & -0.08 & 0.06 & $-0.15^{\star \star \star}$ & $-0.36^{\star \star \star}$ & $0.27^{\star \star \star}$ & \\
\hline ES & $0.17^{\star \star \star}$ & $0.25^{\star \star \star}$ & $0.17^{\star \star \star}$ & $-0.36^{\star \star \star}$ & $-0.29^{\star \star \star}$ & $-0.18^{\star \star \star}$ & $0.19^{\star \star \star}$ & $-0.23^{\star \star \star}$ \\
\hline
\end{tabular}

Abbreviations of traits are explained in Materials and Methods.

Significances were calculated at levels of ${ }^{*} p<0.05$, ${ }^{* \star} p<0.01$ and ${ }^{* \star *} p<0.001$. 
and SL2012 (Table 1). However, only small to medium correlations $(0.24<r<0.63)$ were calculated between the two seed lots. Strongest correlations were found between MGT, T50 and GR36, with I r I > 0.9, and between VI and IS ( $\mathrm{r}$ I $>0.7$ ). Correlations with $0.3 \leq \mathrm{I} \mathrm{r} \mathrm{I} \leq 0.5$ were observed between FG and

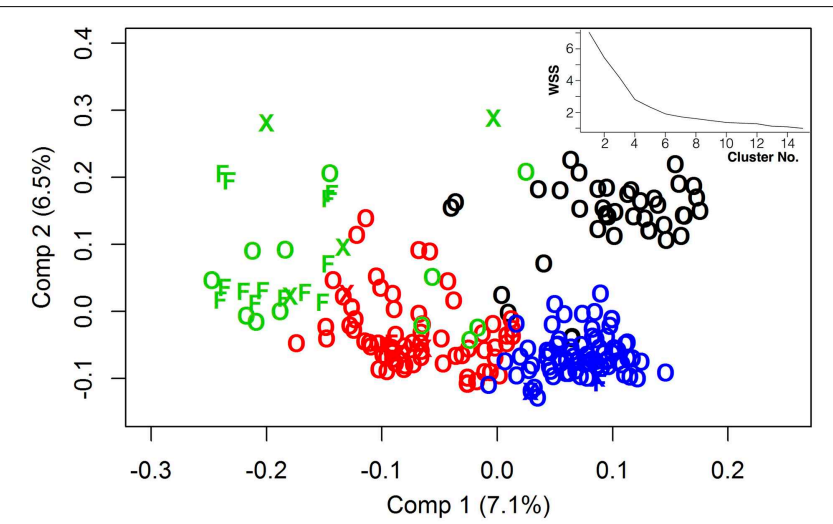

FIGURE 1 | Genetic relatedness of $\mathbf{2 1 8}$ genetically diverse winter-rapeseed lines shown in a principal component analysis regarding the first $\mathbf{2}$ components. Different clusters were represented by different colors. Winter oilseed rape lines were shown as "O," winter fodder lines as "F" and others as "X." Top right: Within-cluster sum of squares depending on the number of clusters applied for k-means clustering. the traits VI, MGT, ES, T50 and GR36, as well as between VI and MGT, T50 and GR36 (Table 2).

With regard to genetic relatedness, the first four PCA components explained $20.98 \%$ of the total genetic variance, while the first two components contributed 7.1\% (Comp 1) and 6.5\% (Comp 2), respectively (Figure 1). Almost all fodder rape genotypes (13 out of 16 ) were assigned to the same cluster (herein referred to as cluster "green"). Taking k-means clusters into account, an overall $F_{S T}$ value of 0.1448 was observed over the whole diversity set, indicating a medium genetic differentiation. Regarding the population pairs, lowest genetic differentiation was found between clusters "red" and "blue" $\left(\mathrm{F}_{\mathrm{ST}}=0.10\right)$, while strongest differentiation was calculated between clusters "green" and "blue" $\left(F_{S T}=0.21\right)$. For all genotype pairs within the diversity set, a mean identity by descent (IBD) of 0.69 was calculated, while values of $0.72,0.73,0.62$, and 0.76 were found within clusters "black," "red," "green," and "blue," respectively (Figure 2). The largest cluster "blue" comprised 79 genotypes and showed the narrowest distribution, whereas the smallest cluster "green" comprised only 29 genotypes but showed the widest distribution of IBD values.

For all chromosomes except C1, C3, C4, and C8, LD decayed to $r^{2}=0.1$ at a distance between marker pairs ranging from $480 \mathrm{kbp}$ (A1) to $1283 \mathrm{kbp}$ (A9) (Figure 3). For chromosomes C3 and C8, $\mathrm{r}^{2}$ did not drop to 0.1 until the distance between marker pairs reached $2014 \mathrm{kbp}$ and $1939 \mathrm{kbp}$, respectively. For chromosome C8, strongly conserved LD was observed for marker

\section{Diversity set}

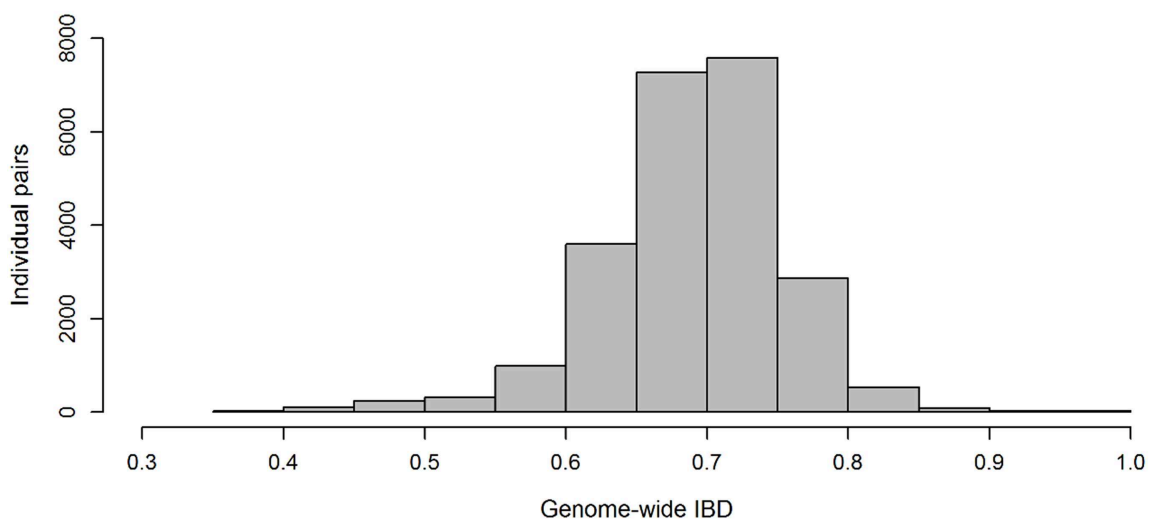

Black

Red

Green

Blue
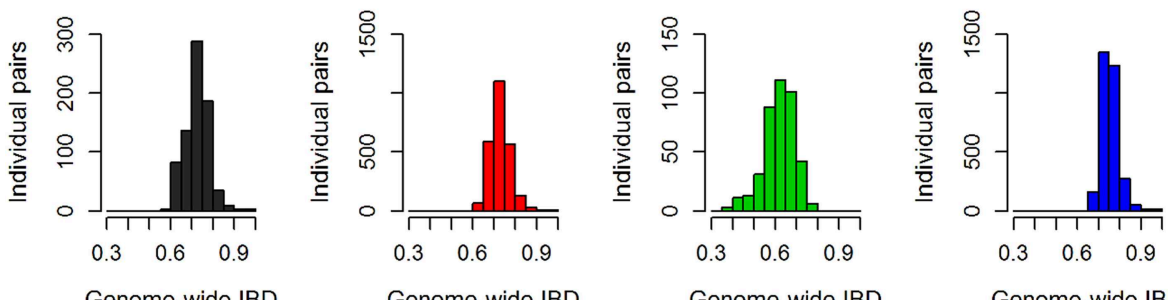

Genome-wide IBD

Genome-wide IBD

FIGURE 2 | Genetic relationship between all individual pairs within the total diversity set $(n=218)$ and within the single clusters from k-means clustering. Histograms show the frequency of individual pairs depending on their kinship coefficients IBD (identity by descent) which score allelic identity by descent. 


\section{Overall LD decay}

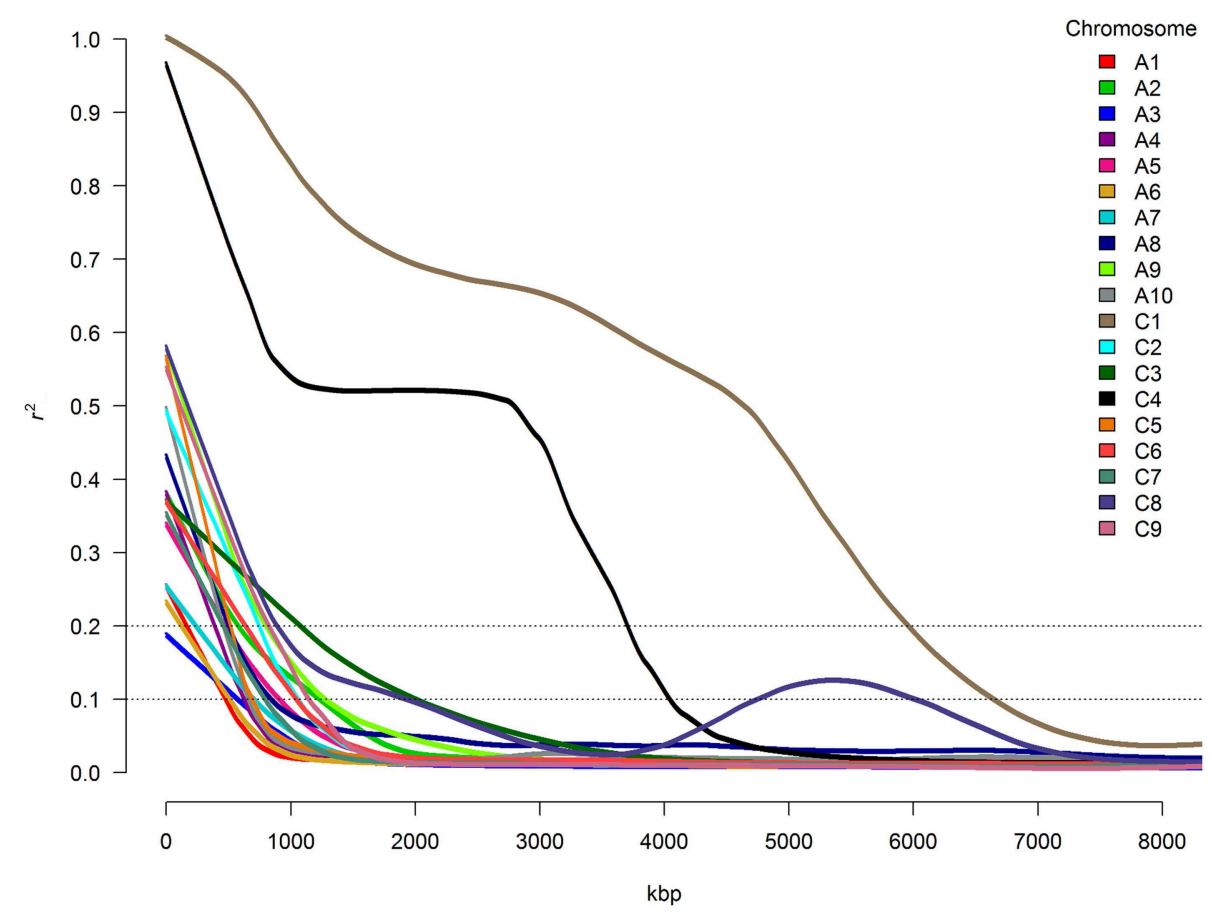

FIGURE 3 | Overall chromosome-wide decay of linkage disequilibrium (LD), shown as smoothed $r^{2}$-values for all marker pairs on each chromosome depending on the distance between marker pairs.

distances between 4500 and $6000 \mathrm{kbp}$. Particularly strong patterns of LD were observed on chromosomes $\mathrm{C} 1$ and $\mathrm{C} 4$, with $r^{2}$-values above 0.1 for up to $6651 \mathrm{kbp}$ and $4048 \mathrm{kbp}$, respectively. The very slow average LD decay on chromosome C4 was mainly determined by the presence of a large conserved LD block localized between 15,429 and 20,449 kbp, whereas conserved LD between 17,787 and $26,635 \mathrm{kbp}$ caused the extremely slow LD decay on chromosome $\mathrm{C} 1$.

Narrow-sense heritability differed considerably among traits and for some traits also between seed lots (Table 3 ). The strongest differences between the 2011 and 2012 seed lots were observed for the trait T50, with relatively low heritability observed within the 2011 seed lot and comparatively high heritability within the 2012 seed low. The traits GR36, ES and TSW showed a medium to high heritability with $h^{2}>0.5$ in all cases. For VI and FG a low heritability was calculated with $h^{2}>0.3$. For GR72 the heritability approached zero for both seed lots. Marker-trait associations that could be confirmed in both seed lots were detected for T50, GR36, GR72, ES, VI, FG, and TSW (Table 3). The observed $\mathrm{F}_{S T}$ value revealed a low to medium genetic differentiation within the diversity set (Figure 2), with subdivision into clusters explaining $14.5 \%$ of the total genetic variation. Calculation of the inflation factor $\lambda$ by genomic control (Devlin and Roeder, 1999; data not shown) confirmed that population structure was predominant. Hence, correction for stratification was performed by mixed-model approach including principal component covariates. Subdivision into clusters caused an increase of IBD values within all clusters except for cluster "green," which mainly comprises fodder rapes and exotic accessions.

\section{Genome-Wide Association Analysis}

The chromosomal regions delineated by haplotype blocks in strong LD $\left(r^{2}>0.4\right)$ with trait-associated markers harbored a total of 681 genes (Table S2). Several genes ascribed to seed germination, seed dormancy and seed and embryo development were disclosed, associated with T50, GR72, ES, FG, and TSW (Table 4). Further investigation showed that none of the candidate genes listed in Table 4 was known to be involved in a mutual pathway. Identical chromosome regions were associated with T50 and GR72, hence the same candidate genes were assumed on chromosomes A9 and C6. The SNP marker Bn-A06-p23586019, associated with TSW, is localized within the non-coding region of the candidate gene BnaA06g34100D. Very strong local LD values $\left(r^{2}>0.8\right)$ were found between markers Bn-A04-p4074166, BnA04-p4217227 and Bn-A04-p10196289, associated with GR72 on chromosome A4, whereas LD with the other surrounding markers was very low $\left(0<r^{2}<0.06\right)$. On the other hand, these markers showed strong extra-chromosomal LD to markers Bn-A03-p5072729 and Bn-A03-p16990947 on chromosome A03, both of which also showed associations with GR72. Although this might be due to co-selection of two epistatically interacting gene loci, this may also suggest a spurious allocation of markers Bn-A04-p4074166, Bn-A04-p4217227 and Bn-A04-p10196289 to 
TABLE 3 | Intervals of linkage disequilibrium (LD) containing significant marker-trait associations for different traits related to germination performance in seeds of an oilseed rape diversity panel produced in Le Rheu, France in 2011 (SL2011) and Asendorf, Germany in 2012 (SL2012), respectively: Chromosome (Chr), Chromosomal position in base pairs [LD interval (bp)], narrow-sense heritability ( ${ }^{2}{ }^{2}$, additive allelic effect (Add. eff.), logarithmic scaled $P$-value $\left(-\log _{10}(p)\right)$.

\begin{tabular}{|c|c|c|c|c|c|c|c|c|}
\hline \multirow[t]{2}{*}{ Trait } & \multirow[t]{2}{*}{ Chr } & \multirow[t]{2}{*}{ LD interval (bp) } & \multicolumn{3}{|c|}{ SL2011 } & \multicolumn{3}{|c|}{ SL2012 } \\
\hline & & & $h^{2}$ & Add. eff. & $-\log _{10}(p)$ & $h^{2}$ & Add. eff. & $-\log _{10}(p)$ \\
\hline \multirow[t]{4}{*}{ TSW } & A1 & $2,094,614-2,132,814$ & 0.84 & -0.23 & 3.28 & 0.76 & -0.23 & 2.65 \\
\hline & A5 & $18,641,259-18,796,724$ & & -0.28 & 2.53 & & -0.35 & 3.11 \\
\hline & A6 & $22,333,133-22,588,108$ & & 0.26 & 5.32 & & 0.19 & 2.95 \\
\hline & C6 & $21,335,770-21,666,697$ & & -0.13 & 2.66 & & -0.15 & 3.07 \\
\hline $\mathrm{VI}$ & C7 & $41,147,197-41,318,742$ & 0.23 & 1.69 & 3.43 & 0.18 & 2.05 & 2.69 \\
\hline$F G$ & $\mathrm{C7}$ & $35,089,636-35,279,703$ & 0.21 & 0.91 & 2.60 & 0.29 & 1.11 & 4.07 \\
\hline \multirow[t]{3}{*}{ T50 } & A9 & $32,206,450-32,410,348$ & 0.34 & 2.76 & 4.80 & 0.86 & 1.98 & 3.06 \\
\hline & C6 & $31,736,330-32,133,403$ & & 1.10 & 2.74 & & 1.23 & 3.85 \\
\hline & C8 & $36,326,419-36,384,818$ & & 1.46 & 2.60 & & 1.41 & 3.02 \\
\hline GR36 & C8 & $36,326,419-3,638,4818$ & 0.50 & -8.36 & 3.47 & 0.77 & -6.64 & 2.76 \\
\hline \multirow[t]{9}{*}{ GR72 } & A3 & $4,478,594-4,515,865$ & 0.07 & -2.01 & 3.16 & 0.00 & -1.78 & 4.28 \\
\hline & & $7,082,702-7,098,892$ & & -1.96 & 2.64 & & -1.82 & 3.69 \\
\hline & & $16,074,818-16,173,009$ & & -2.44 & 3.43 & & -2.01 & 4.22 \\
\hline & A4 & $4,102,377-4,144,135$ & & -2.37 & 3.41 & & -2.01 & 4.04 \\
\hline & & $4,260,229-4,302,660$ & & -2.21 & 3.13 & & -1.99 & 4.33 \\
\hline & & $11,328,102-11,529,657$ & & -2.17 & 3.03 & & -1.79 & 3.59 \\
\hline & A9 & $32,206,450-3,241,0348$ & & -3.00 & 3.00 & & -2.86 & 3.16 \\
\hline & C6 & $31,736,330-32,133,354$ & & -1.59 & 3.10 & & -1.06 & 2.58 \\
\hline & C9 & $42,980,135-43,015,797$ & & -1.89 & 2.70 & & -1.91 & 4.68 \\
\hline \multirow[t]{5}{*}{ ES } & A9 & $6,512,728-7,122,368$ & 0.62 & -0.04 & 2.75 & 0.72 & -0.05 & 4.22 \\
\hline & A10 & $11,631,861-11,677,068$ & & -0.07 & 3.00 & & -0.08 & 3.14 \\
\hline & & $16,085,669-16,164,217$ & & 0.05 & 3.15 & & 0.05 & 3.00 \\
\hline & $\mathrm{C} 2$ & $12,742,547-14,316,723$ & & 0.04 & 2.63 & & 0.04 & 3.37 \\
\hline & C6 & $25,600,351-25,671,165$ & & 0.04 & 2.68 & & 0.06 & 4.73 \\
\hline
\end{tabular}

Abbreviations of traits are explained in Materials and Methods.

the wrong chromosome, hence they were excluded from further analysis.

Two of three particularly promising candidate genes were associated with blocks of strong LD containing QTL for different traits on chromosomes A9 (two independent QTL) and A10. The first of these three, BnaA09g48160D, is located on chromosome A9 only $80.789 \mathrm{kbp}$ from marker Bn-A09-p35262679, which shows significant associations to $\mathrm{T} 50\left(-\log _{10}(\mathrm{p})=4.8\right.$ and 3.1 and GR72 $\left(-\log _{10}(\mathrm{p})=3.2\right.$ and 3.0), but exhibits almost no LD to its nearest flanking markers (Tables 3, 4, Figure 4). BnaA09g48160D encodes for an ortholog of $A$. thaliana gene ARABIDOPSIS TWO-COMPONENT RESPONSE REGULATOR (ARR4). In another independent association peak between 6.51 and $7.12 \mathrm{Mbp}$ on chromosome A9 we localized the gene BnaA09g12770D, between two adjacent markers with very strong $\mathrm{LD}$ that both show significant associations to ES (Tables 3, 4, Figure 5). The SNP marker Bn-A09-p6021939 (associated with $-\log _{10}(\mathrm{p})=4.1$ and 2.8 to ES) is located $141.051 \mathrm{kbp}$ from BnaA09g12770D, which codes for an ortholog of the A. thaliana gene SNOWY COTYLEDON 1 (SCO1). Another significant association peak for ES was localized on chromosome A10 (Tables 3, 4). This region harbors an LD block containing four markers (Bn-A10-p15378551, Bn-A10p15372875, Bn-A10-p15367240, and Bn-A10-p15361519) the latter of which lies directly adjacent $(9.701 \mathrm{kbp})$ to the gene BnaA10g24850D (Figure 6). BnaA10g24850D is an ortholog of another $A$. thaliana gene implicated in germination and early development, ARGINYL-t-RNA PROTEIN TRANSFERASE 1 (ATE1).

Pigment concentrations were measured in the cotyledons of $5 \mathrm{~d}$ old seedlings from the two genotype bulks, featuring consistently low and high ES values, respectively. Mean concentrations of chlorophyll $\mathrm{a}$ and $\mathrm{b}$ were found to be significantly lower in genotypes with poor performance in ES (Figure 7).

Marker haplotypes for all disclosed regions associated with T50 and ES are displayed in Figure 8, which compares the most frequent locus-specific haplotypes with favorable and undesirable effects on trait performance. Because a threshold of $r^{2}>0.4$ was chosen for LD block definition, a smaller fraction of recombinant haplotypes also occurred. A list of all observed haplotypes and their frequencies is provided in Table S3. With increasing number of favorable alleles, performance is shown to be improved for both T50 and ES (Figure 9). For T50 an increase in the number of undesirable haplotypes was associated with a decrease in 
TABLE 4 | List of candidate genes ascribed to seed germination, dormancy and seed development: Chromosome (Chr), Name of the candidate gene (Candidate gene) and absolute chromosomal position in base pairs [Position (bp)].

\begin{tabular}{lccc}
\hline Trait & Chr & Candidate gene & Position (bp) \\
\hline TSW & A6 & BnaA06g34100D & $22,551,497-22,558,918$ \\
& & BnaA06g33970D & $22,490,437-22,491,055$ \\
& & BnaA06g33830D & $22,421,795-22,424,461$ \\
& & BnaA06g33880D & $22,446,550-22,447,630$ \\
& C6 & BnaC06g19160D & $21,499,780-21,506,256$ \\
FG & C7 & BnaC07g31020D & $35,273,679-35,275,807$ \\
T50 & A9 & BnaA09g48160D & $32,313,276-3,231,4748$ \\
& C6 & BnaC06g31150D & $31,787,778-31,792,013$ \\
GR72 & A9 & BnaA09g48160D & $32,313,276-32,314,748$ \\
& C6 & BnaC06g31150D & $31,787,778-31,792,013$ \\
& C9 & BnaC09g40630D & $42,994,450-42,995,360$ \\
& & BnaC09g40640D & $42,997,059-42,998,540$ \\
ES & A9 & BnaA09g12770D & $6,802,082-6,804,959$ \\
& & BnaA09g12820D & $6,856,835-6,858,406$ \\
& & BnaA09g12800D & $6,834,031-6,835,814$ \\
& & BnaA09g13040D & $7,099,948-7,100,930$ \\
& & BnaA10g24850D & $16,143,505-16,146,463$ \\
& A10 & BnaA10g24780D & $16,125,049-16,127,616$
\end{tabular}

Abbreviations of traits are explained in Materials and Methods.

the number of individuals per bulk (Table S3). While the main fraction of lines $(n=133)$ exhibited the favorable haplotype at all three loci, a combination of all three unfavorable alleles was observed in only one line. For ES, neither a combination of all favorable, nor all undesirable haplotypes occurred, with most individuals ( $n=105)$ exhibiting 2 favorable alleles (Table S3).

\section{Discussion}

\section{The Key Elements of Seed Germination}

Seed germination has a decisive influence on homogeneous field emergence and successful seedling establishment and is therefore a basic target for the development of vigorous crop plants with stable yield performance. According to Finch-Savage et al. (2010), germination speed is a major key element of vigorous seeds, along with a rapid initial downward and upward seedling growth.

While traits like seed imbibition speed (IS) and seed volume increase (VI) can be used for characterization of initial water uptake within the first hours after soaking, mean germination time (MGT), time to reach $50 \%$ of germination (T50), germination rate within $36 \mathrm{~h}$ (GR36) and first germination (FG) provide insight into the speed of the total germination process until the radicle breaks through the testa. Elongation speed of the radicle (ES) is not part of the germination process per se, but is an important component of pre-emergence seedling growth after entry into the third phase of water uptake (Bewley, 1997). Although seed vigor is constituted by both germination speed and preemergence seedling growth, these two criteria are presumed to contribute independently to seed vigor performance (Dutt and

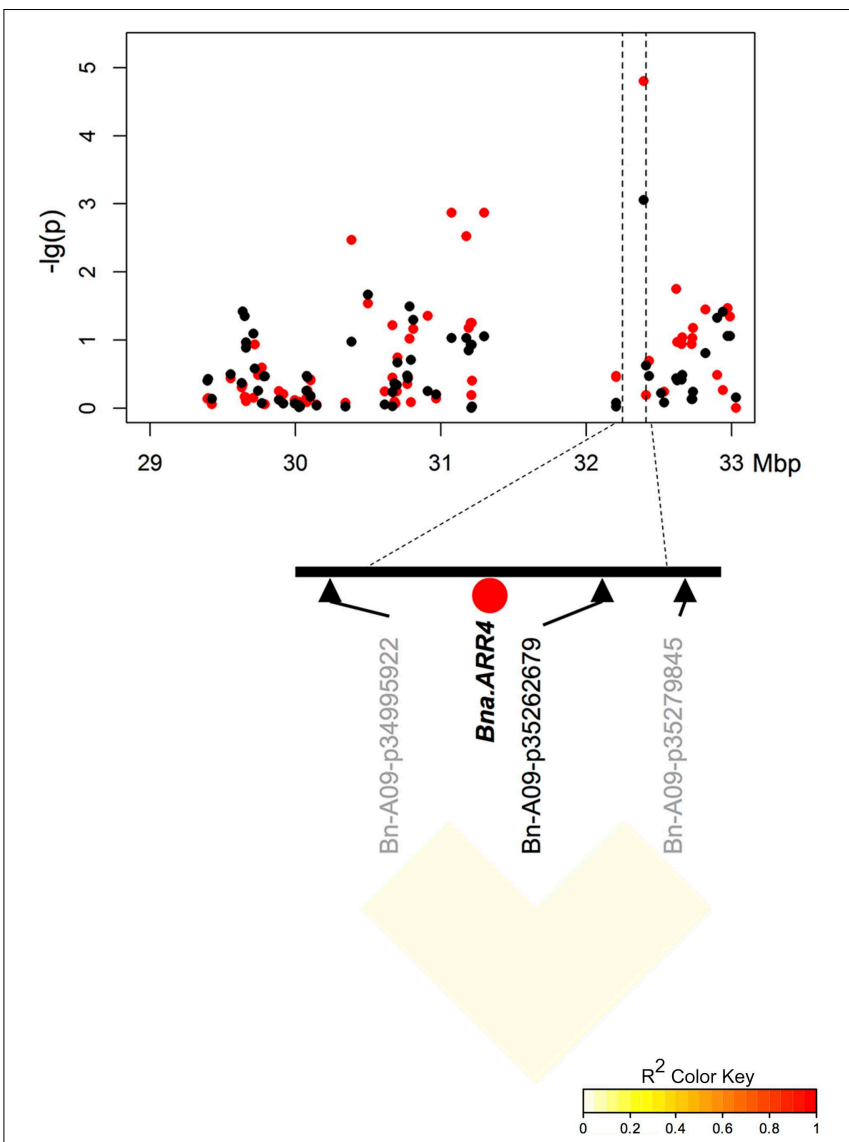

FIGURE 4 | Manhattan plot describing marker-trait associations for the trait ES within the chromosomal region around candidate BnaA09g48160D (Bna.ARR4) on chromosome A9, and correlations between surrounding markers. Red dots represent associations calculated for SL2011. Black dots represent associations for SL2012.

Geneve, 2007). This assumption is strengthened by the very low or absent phenotypic correlations we observed between ES and germination speed parameters (FG, MGT, T50, GR36) in the $B$. napus diversity panel. Interestingly, we found no evidence for a decisive influence of seed weight (TSW) on germination performance, absolute germination rate or radicle growth, with only extremely low correlations being observed to these parameters. This is in accordance to Bettey et al. (2000), who attributed no influence of seed weight to mean germination time or absolute germination in Brassica oleracea. On the other hand, Hanumaiah and Andrews (1973) described a positive relationship between seed size and seedling growth for B. rapa and B. oleracea. According to our observations TSW should be regarded as a seed yield parameter in oilseed rape rather than a contributing factor for germination performance.

Weak correlations between germination speed (T50, MGT, GR36, and FG) and seed imbibition in terms of VI and IS indicate that the velocity of total germination is impacted only to a limited extent by initial water uptake. In turn, induction of the biochemical processes contributing to embryo expansion and leading to visible germination seem to be mainly independent 


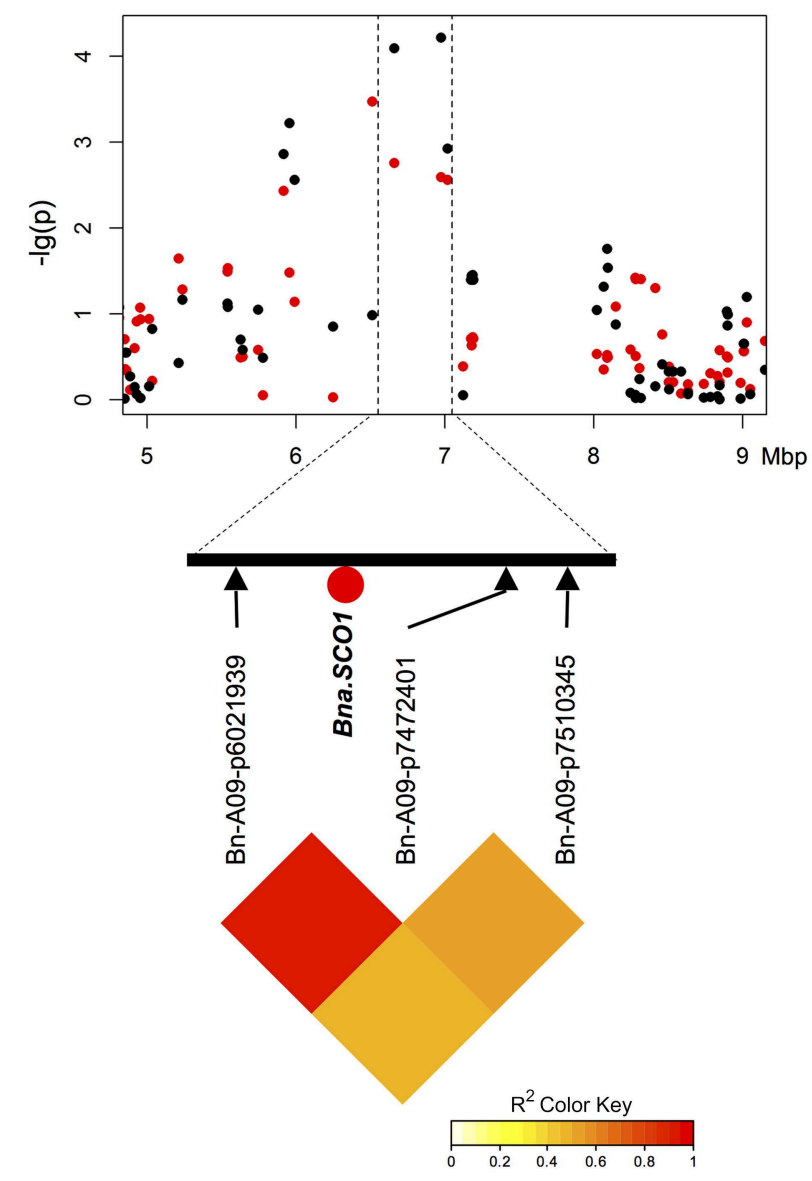

FIGURE 5 | Manhattan plot describing marker-trait associations for the trait T50 within the chromosomal region around candidate BnaA09g12770D (Bna.SCO1) on chromosome A9, and correlations between the associated marker and both flanking markers. Red dots represent associations calculated for SL2011. Black dots represent associations for SL2012.

from imbibition speed. Apparently other factors induce the release of germination, and in this context it is worthwhile to consider seed dormancy. According to Finch-Savage and LeubnerMetzger (2006), seed dormancy is an innate seed property that defines the environmental conditions that must be met before the seed can germinate. With respect to the diverse regulation levels, dormancy can be divided into different dormancy types (Baskin and Baskin, 2004). For members of the Brassicaceae it is assumed that mainly physiological dormancy is predominant (Müller et al., 2006). Our results indicate that differences in germination speed in $B$. napus are not solely dependent on water availability or the presence of favorable conditions for the induction of biochemical actions contributing to seed germination. In fact, the observed variation in germination speed might be caused by differences in the manifestation of seed dormancy. However, because the in vitro germination assay we applied implements optimal and controlled germination conditions, two explanations are possible: On the one hand, the diversity panel we investigated appears to exhibit differences in genetically determined responsiveness to dormancy release factors. On the other hand,

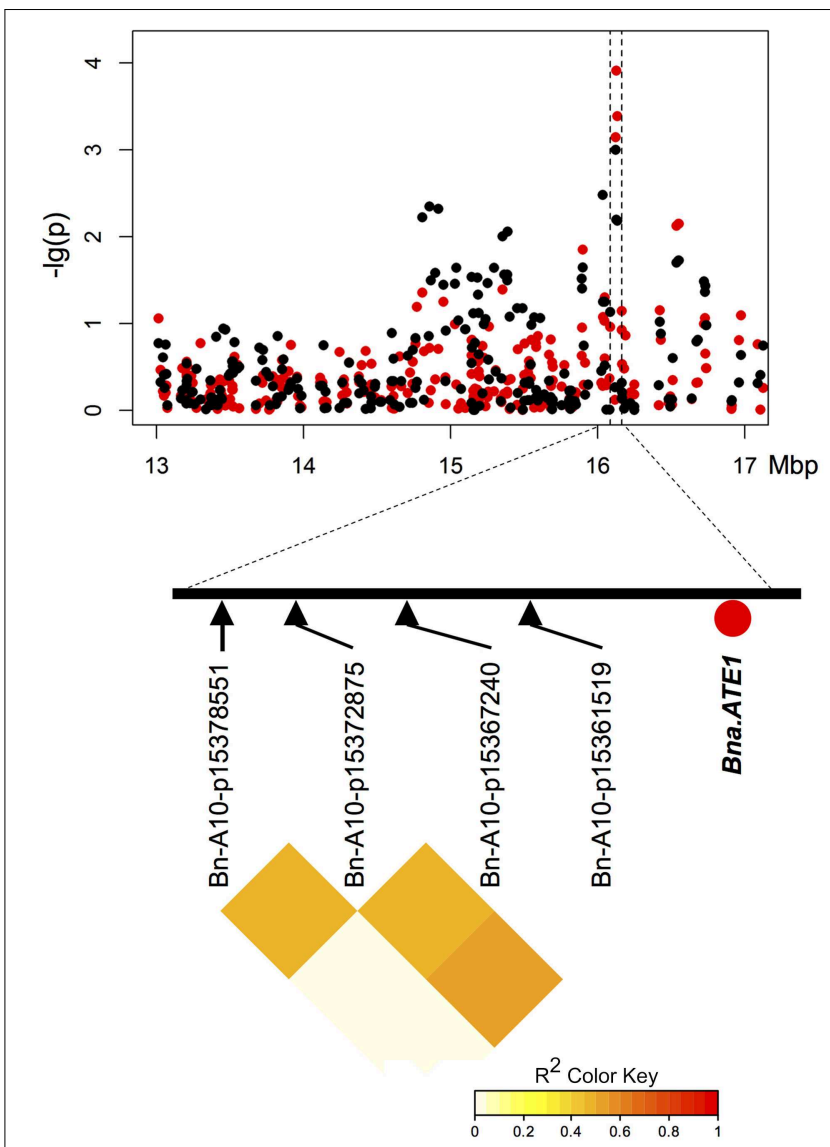

FIGURE 6 | Manhattan plot describing marker-trait associations for the trait ES for the chromosomal region around candidate $B$ naA10g24850D (Bna.ATE1) on chromosome A10, and correlations between markers of the linkage group harboring BnaA10g24850D. Red dots represent associations calculated for SL2011. Black dots represent associations for SL2012.

the observed differences in germination performance also appear to be strongly determined by the conditions predominant during and after seed production. The latter hypothesis is strengthened by the observation of only moderate correlations between the two seed lots (Table 1). As both seed lots were tested for germination under constant laboratory conditions, the observed differences in performance between the two seed lots must reflect genotype by environment interactions during seed ripening in the field and/or during the subsequent seed storage. One possible mechanism could be epigenetic imprinting during seed development due to stress conditions in the maternal environment.

\section{Exploring the Genetic Basis of Seed Germination and Seedling Growth}

Against the background of a strong environmental dependence of seed germination and early seedling growth, breeders are interested in the fixed fraction of variables influencing seed vigor performance. Although the physiology of seed germination and dormancy is well studied and extensively described in literature (e.g., reviewed in Bewley, 1997; FinchSavage and Leubner-Metzger, 2006; Finkelstein et al., 2008; Rajjou et al., 2012), there is still a lack of knowledge about 
individual genes involved in these complex traits, particularly in crop species. Both germination and dormancy underlie polygenic control that can only be elucidated by quantitative genetics approaches.

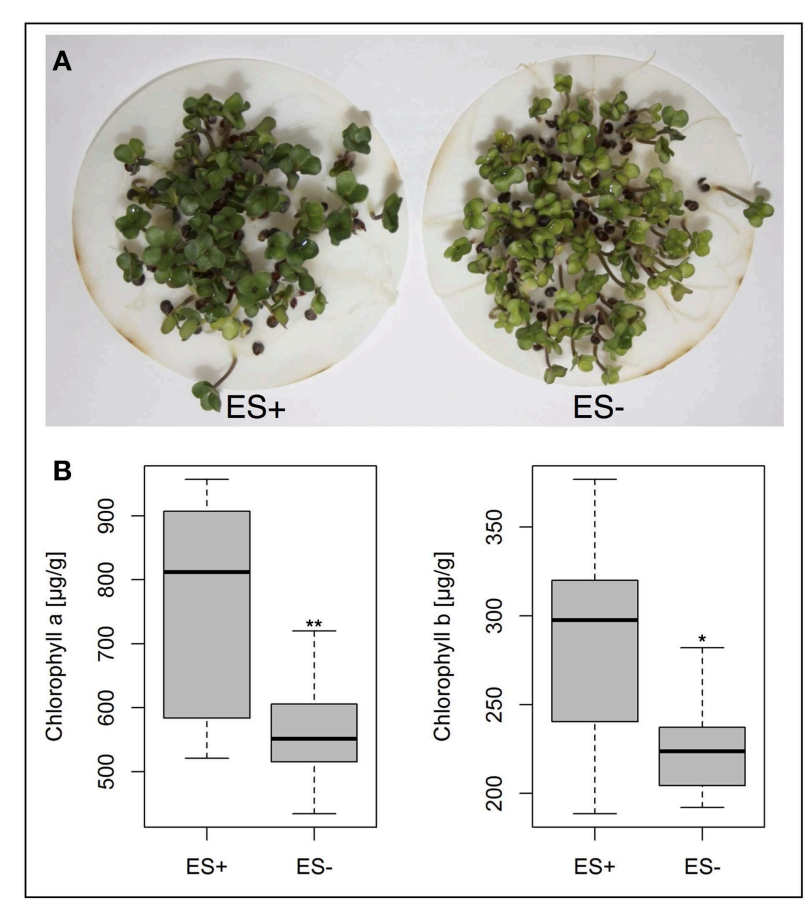

FIGURE 7 | (A) Phenotypic variation for cotyledon chlorophyll pigmentation in $B$. napus genotypes showing high (ES+) and low (ES-) elongation radicle rates. (B) Box plots representing chlorophyll a and chlorophyll b concentrations of 10 genotypes each, showing high and low elongation radicle rates. Significant differences were calculated with student's $t$-test at $p<0.05^{\star}$ and $p<0.01^{\star *}$
To estimate how much genotypic variation contributes to the observed phenotypic variation we calculated narrow-sense heritability $\left(\mathrm{h}^{2}\right)$, which captures only that part of genetic variation caused by additive genetic values (Visscher et al., 2008). Narrowsense heritability can only be calculated based on the detected associations, so that undetected associations may repress $h^{2}$ values. The observed high $h^{2}$-values for TSW agree with findings from earlier studies (Basunanda et al., 2010; Fan et al., 2010; Khan et al., 2013; Xing et al., 2014). In contrast, very low $h^{2}$-values for GR72 suggested that the absolute germination rate is almost completely dependent on the environmental state and can hardly be influenced by selection. Nevertheless, identical associations for T50 and GR72 on chromosomes A9 and C6 indicate that both germination speed and absolute germination rate seem to be controlled by the same genetic factors (see Table 3), so that selection for T50 may also lead to improvement of GR72.

The candidate gene disclosure strategy we applied accounted for trait associations that were detectable in both of the investigated seed lots. The use of local LD to delineate regions of interest around significantly associated SNP markers proved a valuable method for defining potential candidate genes. The very strong LD on some C-sub-genome chromosomes corresponds to findings in other B. napus gene pools and reflects extreme selection for specific seed quality traits (Li et al., 2014; Qian et al., 2014). Strong LD conservation can complicate candidate gene identification from association peaks, hence it is important to consider local LD when defining QTL confidence intervals for candidate gene selection. The validity of the trait associations we detected appears to be confirmed by the identification of comparatively small regions of strong LD that harbor highly interesting genes involved in processes closely related to seed germination and seedling development.

Several genes ascribed to seed germination, dormancy, seed development and radicle emergence were identified within the associated regions (Table 4). For example, the $A$. thaliana gene

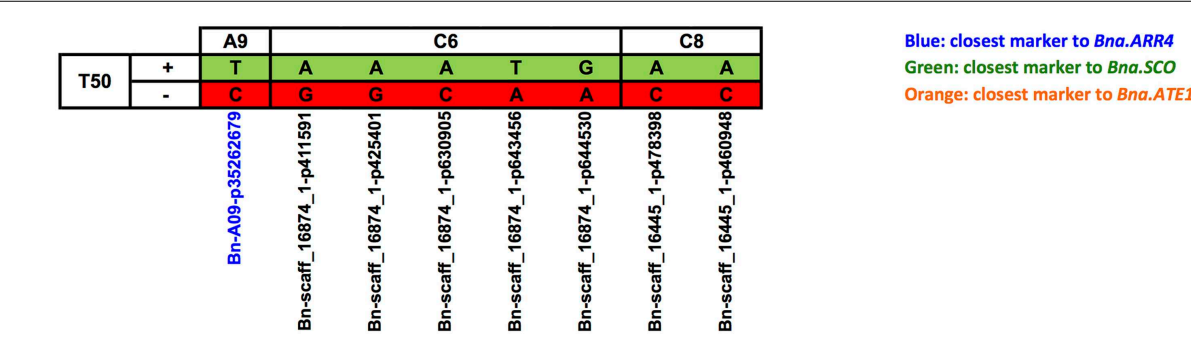

\begin{tabular}{|c|c|c|c|c|c|c|c|c|c|c|c|c|c|c|c|c|c|c|c|c|c|c|}
\hline \multirow{3}{*}{ ES } & & \multicolumn{3}{|c|}{ A9 } & \begin{tabular}{|l|} 
A10-1 \\
\end{tabular} & \multicolumn{3}{|c|}{ A10-2 } & \multicolumn{13}{|c|}{ C2 } & \multirow{2}{*}{$\begin{array}{c}\text { C6 } \\
G\end{array}$} \\
\hline & + & A & C & G & $T$ & G & A & $\mathrm{T}$ & G & G & A & C & A & A & $\mathrm{T}$ & G & A & G & $\mathrm{T}$ & A & $\mathbf{T}$ & \\
\hline & - & G & A & A & C & A & G & C & $T$ & $T$ & G & $T$ & G & G & C & $\mathrm{T}$ & G & A & G & G & G & A \\
\hline & & 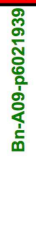 & 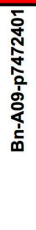 & 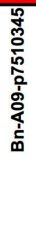 & 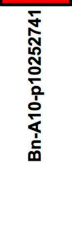 & 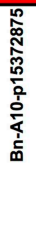 & 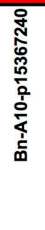 & 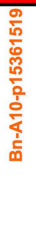 & 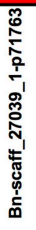 & 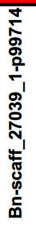 & 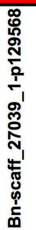 & 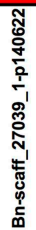 & 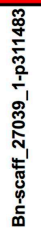 & 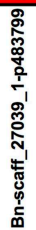 & 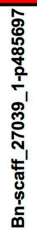 & 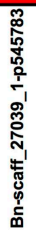 & 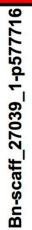 & 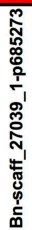 & 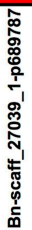 & 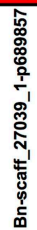 & 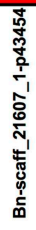 & 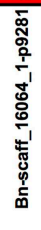 \\
\hline
\end{tabular}

FIGURE 8 | Loci-specific alleles of single SNP markers and different haplotypes from LD-blocks harboring various SNP markers associated with T50 and ES. Favorable alleles $(+)$ were marked in green, alleles with negative effect on trait performance (-) were shown in red. Corresponding marker names were listed below. T50, Time to reach 50\% of germination; ES, Elongation speed of the radicle. 

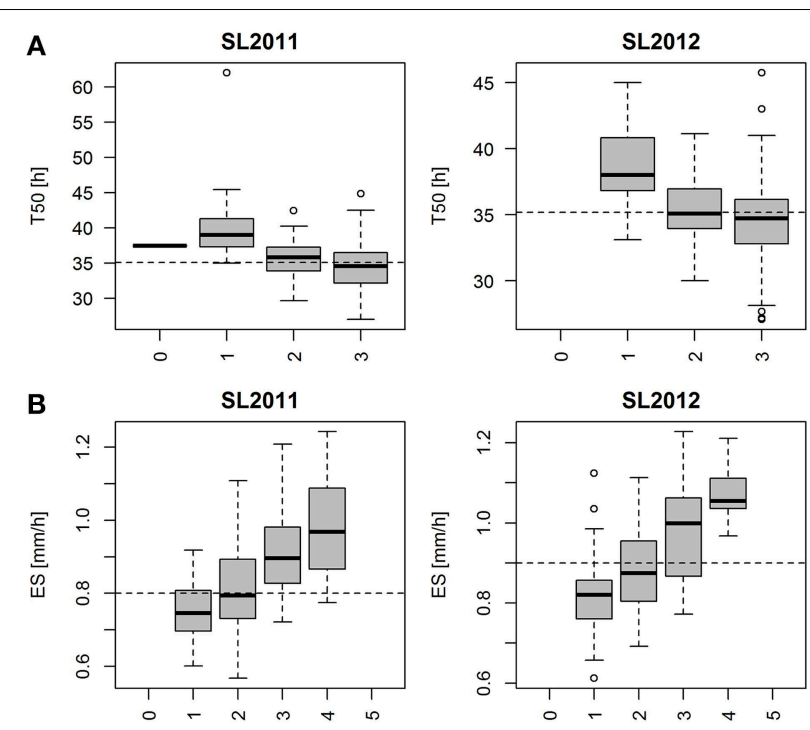

FIGURE 9 | (A) Boxplot representing the phenotypic distribution for T50 (Time to reach 50\% of germination) in two different seed lots, produced in 2011 (SL2011) and 2012 (SL2012). Each whisker represents a bulk of genotypes exhibiting no favorable haplotype (0), one favorable haplotype (1), two favorable haplotypes (2) and three favorable haplotypes (3) regarding the three associated regions on chromosome A9, C6 and C8. Population means were indicated as dashed lines. (B) Boxplot representing the phenotypic distribution for ES (Elongation speed of the radicle) in two different seed lots, produced in 2011 (SL2011) and 2012 (SL2012). Each whisker represents a bulk of genotypes exhibiting no favorable haplotype (0), one favorable haplotype (1), two favorable haplotypes (2) three favorable haplotypes (3), four favorable haplotypes (4) and five favorable haplotypes (5) regarding the five disclosed associated regions on chromosome A9, A6, A10, C2, and C6. Population means were indicated as dashed lines.

$A R R 4$ is a cytokinin-dependant antagonistic regulator of transcription activator $A B I 5$, which is directly involved in the ABA mediated regulation of seed germination (Wang et al., 2011). The presence of a Bn.ARR4 homolog in a region of LD with an association peak for T50 and GR72 corresponds to the expected role of ABA-mediated dormancy and germination release. In A. thaliana the gene SCO1 was found to encode a plastidic translation elongation factor $G$, which is reported to be essential for eoplast to chloroplast transition during early germination (Ruppel and Hangarter, 2007). Furthermore, A. thaliana scol mutations also cause a chlorophyll deficiency resulting in pale-green to white coloring of seedling cotyledons, and the seedling chlorophyll deficiency of scol mutants is coupled with a delayed seed germination and development (Albrecht et al., 2006). Accordingly, B. napus genotypes with extreme segregation for ES also showed significant differences in seedling chlorophyll content. Associations of Bna.SCO1 haplotypes to ES, together with the corresponding chlorophyll phenotypes, support a causal role for this gene in the speed of early radicle growth in $B$. napus. Mutations in ATE genes encoding for arginyl-t-rna protein transferases have also been shown to cause reductions in A. thaliana seedling root growth (Holman et al., 2009). Allelic differences in Bn.ATE1 within LD haplotypes associated with ES are hence also strong positional and functional candidates for control of radicle growth during and after germination.

Genotypic classification for T50 and ES revealed that both traits can potentially be improved by pyramiding of favorable alleles (Figures 9A,B). For both traits the absence among the diversity panel of individuals with three (T50) or five (ES) unfavorable haplotypes indicates that strong natural and artificial selection has occurred in cultivated forms of $B$. napus against individuals with very poor germination and vigor. Consequently, effects of loci associated with these traits might be underestimated because specific undesirable allele combinations are absent, thus restricting the extent of phenotypic variability within the diversity set. For ES we also observed a lack of favorable haplotype combinations, indicating that early seedling growth could be substantially improved beyond the phenotypic diversity in the present study.

\section{Conclusions}

Large-scale automated phenotyping revealed a broad phenotypic variability in germination performance across a $B$. napus diversity panel. As expected, narrow-sense heritability revealed that germination performance is influenced by both genetic as well as environmental factors. As the latter were constant during in vitro phenotyping, the observed differences in germination performance between two different seed lots could be attributed to the conditions predominant during seed production and storage. No relationship of germination traits to seed weight could be detected. Instead, the results indicate that genetic determinants for the manifestation of seed dormancy may be a decisive factor influencing inheritance of germination performance in $B$. napus. In a genome-wide association analysis several promising genes, including Bna.SCO1, Bna.ARR4, and Bna.ATE1, were found within regions showing LD with QTL for seed germination and vigor. Combination of positive alleles for the loci we identified should facilitate a decisive increase in germination performance and early seedling growth in oilseed rape, improving prospects for breeding of these complex, poorly selectable traits. As such the study provides a basis for the establishment of genomic selection tools for improved seed germination and seed vigor in rapeseed.

\section{Author Contributions}

SH and RS designed the study and interpreted the results. RS, $\mathrm{NN}, \mathrm{AA}$, and GL developed the diversity panel and generated seed lots for the phenotypic analysis. NN, AA, GL, and FB performed field phenotyping and analyzed the field phenotype data, SD and MW performed the automated germination phenotyping, and FB generated the genome-wide SNP data. SH and MF performed the GWAS analysis. SH and RS wrote the manuscript and all authors corrected and approved the final version.

\section{Acknowledgments}

The work described in this manuscript was performed within the framework of the transnational PLANT-KBBE cooperation 
project CONVIGOUR, with funding from BMBF (Germany) and ANR (France). We thank Deutsche Saatveredelung AG (Lippstadt, Germany) and INRA-IGEPP (Le Rheu, France) for generation of seed materials and Sarah Schiessl and Kai Voss-Fels for helpful discussions and biostatistics support.

\section{References}

Albrecht, V., Ingenfeld, A., and Apel, K. (2006). Characterization of the Snowy Cotyledon 1 mutant of Arabidopsis thaliana: the impact of chloroplast elongation factor $\mathrm{G}$ on chloroplast development and plant vitality. Plant Mol. Biol. 60, 507-518. doi: 10.1007/s11103-005-4921-0

Aulchenko, Y. S., Ripke, S., Isaacs, A., and van Duijn, C. M. (2007). GenABEL: an R library for genome-wide association analysis. Bioinformatics 23, 1294-1296. doi: 10.1093/bioinformatics/btm108

Baskin, J. M., and Baskin, C. C. (2004). A classification system for seed dormancy. SSR 1, 1-16. doi: 10.1079/SSR2003150

Basunanda, P., Radoev, M., Ecke, W., Friedt, W., Becker, H. C., and Snowdon, R. J. (2010). Comparative mapping of quantitative trait loci involved in heterosis for seedling and yield traits in oilseed rape (Brassica napus L.). Theor. Appl. Genet. 120, 271-281. doi: 10.1007/s00122-009-1133-Z

Bettey, M., Finch-Savage, W. E., King, G. J., and Lynn, J. R. (2000). Quantitative genetic analysis of seed vigour and pre-emergence seedling growth traits in Brassica oleracea. New Phytol. 148, 277-286. doi: 10.1046/j.14698137.2000.00760.x

Bewley, J. D. (1997). Seed germination and dormancy. Plant Cell 9, 1055-1066. doi: 10.1105/tpc.9.7.1055

Chalhoub, B., Denoeud, F., Liu, S., Parkin, I. A. P., Tang, H., Wang, X., et al. (2014). Early allopolyploid evolution in the post-Neolithic Brassica napus oilseed genome. Science 345, 950-953. doi: 10.1126/science.1253435

Conesa, A., Götz, S., Garcia-Gomez, J. M., Terol, J., Talon, M., and Robles, M. (2005). Blast2GO: a universal tool for annotation, visualization and analysis in functional genomics research. Bioinformatics 21, 3674-3676. doi: 10.1093/bioinformatics/bti610

Demilly, D., Ducournau, S., Wagner, M. H., and Dürr, C. (2014). “Digital imaging of seed germination," in Plant Image Analysis-Fundamentals and Applications, eds S. Dutta Gupta and Y. Ibaraki (Boca Raton: CRC Press), 147-163.

Devlin, B., and Roeder, K. (1999). Genomic control for association studies. Biometrics 55, 997-1004. doi: 10.1111/j.0006-341X.1999. 00997.x

Ducournau, S., Feutry, A., Plainchault, P., Revollon, P., Vigouroux, B., and Wagner, M. H. (2004). An image acquisition system for automated monitoring of the germination rate of sunflower seeds. Comput. Electron. Agric. 44, 189-202. doi: 10.1016/j.compag.2004.04.005

Ducournau, S., Feutry, A., Plainchault, P., Revollon, P., Vigouroux, B., and Wagner, M. H. (2005). Using computer vision to monitor germination time course of sunflower (Helianthus annuus L.) seeds. Seed Sci. Technol. 33, 329-340. doi: 10.15258/sst.2005.33.2.06

Dutt, M., and Geneve, R. (2007). Time to radicle protrusion does not correlate with early seedling growth in individual seeds of impatiens and petunia. J. Am. Soc. Hortic. Sci. 132, 423-428. Available online at: http://www.crec.ifas.ufl.edu/academics/faculty/dutt/pdfs/Time_to_radicle_pro trusion.pdf

Edwards, D., Batley, J., and Snowdon, R. J. (2013). Accessing complex crop genomes with next-generation sequencing. Theor. Appl. Genet. 126, 1-11. doi: 10.1007/s00122-012-1964-X

Fan, C., Cai, G., Qin, J., Li, Q., Yang, M., Wu, J., et al. (2010). Mapping of quantitative trait loci and development of allele-specific markers for seed weight in Brassica napus. Theor. Appl. Genet. 121, 1289-1301. doi: 10.1007/s00122-010$1388-4$

Finch-Savage, W. E., Clay, H. A., Lynn, J. R., and Morris, K. (2010). Towards a genetic understanding of seed vigour in small-seeded crops using natural variation in Brassica oleracea. Plant Sci. 179, 582-589. doi: $10.1016 /$ j.plantsci.2010.06.005

\section{Supplementary Material}

The Supplementary Material for this article can be found online at: http://www.frontiersin.org/journal/10.3389/fpls.2015. 00221/abstract

Finch-Savage, W. E., and Leubner-Metzger, G. (2006). Seed dormancy and the control of germination. New Phytol. 171, 501-523. doi: 10.1111/j.14698137.2006.01787.x

Finkelstein, R., Reeves, W., Ariizumi, T., and Steber, C. (2008). Molecular aspects of seed dormancy. Annu. Rev. Plant Biol. 59, 387-415. doi: 10.1146/annurev.arplant.59.032607.092740

Foolad, M. R., Lin, G. Y., and Chen, F. Q. (1999). Comparison of QTLs for seed germination under non-stress, cold stress and salt stress in tomato. Plant Breed. 118, 167-173. doi: 10.1046/j.1439-0523.1999.118002167.x

Friedt, W., and Snowdon, R. (2010). "Oilseed rape," in Oil Crops Handbook of Plant Breeding, Vol. 4, eds J. Vollmann and I. Rajcan (New York, NY: Springer), 91-126.

Hanumaiah, L., and Andrews, C. H. (1973). Effects of seed size in cabbage and turnip on performance of seeds, seedling size and plants. Proc. Assoc. Off. Seed Anal. 63, 117-125.

Hatzig, S., Zaharia, L. I., Abrams, S., Hohmann, M., Legoahec, L., Bouchereau, A., et al. (2014). Early osmotic adjustment responses in drought-resistant and drought-sensitive oilseed rape. J. Intergr. Plant Biol. 56, 797-809. doi: 10.1111/jipb.12199

Holman, T. J., Jones, P. D., Russel, L., Medhurst, A., Úbeda Tomás, S., Talloji, P., et al. (2009). The $\mathrm{N}$-end rule pathway promotes seed germination and establishment through removal of ABA sensitivity in Arabidopsis. Proc. Natl. Acad. Sci. U.S.A. 11, 4549-4554. doi: 10.1073/pnas.0810280106

Khan, M. H., Bhuiyan, S. R., Rashid, M. H., Gosh, S., and Paul, S. K. (2013). Variability and heritability analysis in short duration and high yielding Brassica rapa L. Bangladesh J. Agril. Res. 38, 647-657. doi: 10.3329/bjar.v38i4.19020

Korte, A., and Farlow, A. (2013). The advantages and limitations of trait analysis with GWAS: a review. Plant Methods 9:29. doi: 10.1186/1746-4811-9-29

Larsen, S., Povlsen, F. V., Eriksen, E. N., and Pedersen, H. C. (1998). The influence of seed vigour on field performance and the evaluation of the applicability of the controlled deterioration vigour test in oil seed rape (Brassica napus) and pea (Pisum sativum). Seed Sci. Technol. 26, 627-641.

Leopold, A. C. (1983). Volumetric components of seed imbibition. Plant Physiol. 73, 677-680. doi: 10.1104/pp.73.3.677

Li, F., Chen, B., Xu, K., Wu, J., Song, W., Bancroft, I., et al. (2014). Genomewide association study dissects the genetic architecture of seed weight and seed quality in rapeseed (Brassica napus L.). DNA Res. 21, 355-367. doi: 10.1093/dnares/dsu002

MacQueen, J. (1967). Some methods for classification and analysis of multivariate observations. Proc. Fifth Berkeley Symp. Math. Statist. Prob. 1, 281-297.

Matthews, S., and Khajeh Hosseini, M. (2006). Mean germination time as an indicator of emergence performance in soil of seed lots of maize. Seed Sci. Technol. 34, 339-347. doi: 10.15258/sst.2006.34.2.09

Matthews, S., Wagner, M. H., Kerr, L., McLaren, G., and Powell, A. A. (2012). Automated determination of germination time courses by image capture and early counts of radicle emergence lead to a new vigour test for winter oilseed rape (Brassica napus). Seed Sci. Technol. 40, 413-424. doi: 10.15258/sst.2012.40.3.12

Müller, K., Tintelnot, S., and Leubner-Metzger, G. (2006). Endospermlimited Brassicaceae seed germination: abscisic acid inhibits embryo-induced endosperm weakening of Lepidium sativum (cress) and endosperm rupture of cress and Arabidopsis thaliana. Plant Cell Physiol. 47, 864-877. doi: $10.1093 / \mathrm{pcp} / \mathrm{pcj} 059$

Nordborg, M., and Weigel, D. (2008). Next-generation genetics in plants. Nature 456, 720-723. doi: 10.1038/nature07629

Obermeier, C., Hossain, M. A., Snowdon, R., Knüfer, J., von Tiedemann, A., and Friedt, W. (2013). Genetic analysis of phenylpropanoid metabolites associated with resistance against Verticillium longisporum in Brassica napus. Mol. Breed. 31, 347-361. doi: 10.1007/s11032-012-9794-8 
Perry, D. A. (1978). Problems of the development and application of vigour tests to vegetable seeds. Acta Hortic. 83, 141-146.

Price, A. L., Zaitlen, N. A., Reich, D., and Patterson, N. (2010). New approaches to population stratification in genome-wide association studies. Nat. Rev. Genet. 11, 459-463. doi: $10.1038 / \mathrm{nrg} 2813$

Qian, L., Qian, W., and Snowdon, R. J. (2014). Sub-genomic variation patterns as a signature of breeding in rapeseed. BMC Genomics. 15:1170. doi: 10.1186/1471-2164-15-1170

Rajjou, L., Duval, M., Gallardo, K., Catusse, J., Bally, J., Job, C., et al. (2012). Seed Germination and Vigor. Annu. Rev. Plant Biol. 63, 507-533. doi: 10.1146/annurev-arplant-042811-105550

Rousset, F. (2008). GENEPOP'007: a complete re-implementation of the GENEPOP software for Windows and Linux. Mol. Ecol. Resour. 8, 103-106. doi: $10.1111 / j .1471-8286.2007 .01931 . x$

Ruppel, N., and Hangarter, R. P. (2007). Mutations in a plastidlocalized elongation factor $\mathrm{G}$ alter early stages of plastid development in Arabidopsis thaliana. BMC Plant Biol. 7:37. doi: 10.1186/14712229-7-37

Schiessl, S. V., Samans, B., Hüttel, B., Reinhard, R., and Snowdon, R. J. (2014). Capturing sequence variation among flowering-time regulatory gene homologs in the allopolyploid crop species. Front. Plant Sci. 5:404. doi: 10.3389/fpls.2014.00404

Svishcheva, G. S., Axenovich, T. I., Belonogova, N. M., van Duijn, C. M., and Aulchenko, Y. S. (2012). Rapid variance components-based method for whole-genome association analysis. Nat. Genet. 44, 1166-1170. doi: 10.1038/ ng. 2410

Visscher, P. M., Hill, W. G., and Wray, N. R. (2008). Heritability in the genomics era-concepts and misconceptions. Nat. Rev. Genet. 9, 255-266. doi: $10.1038 / \operatorname{nrg} 2322$
Wagner, M. H., Demilly, D., Ducournau, S., Dürr, C., and Léchappé, J. (2011). Computer vision for monitoring seed germination from dry state to young seedlings. Seed Test. Int. 142, 49-51. Available online at: http://prodinra.inra. fr/record $/ 47502$

Wang, Y., Li, L., Ye, T., Zhao, S., Liu, Z., Feng, Y. Q., et al. (2011). Cytokinin antagonizes ABA suppression to seed germination of Arabidopsis by downregulating ABI5 expression. Plant J. 68, 249-261. doi: 10.1111/j.1365-313X.2011.04683.x

Wellburn, A. R. (1994). The spectral determination of chlorophylls a and b, as well as total carotenoids, using various solvents with spectrophotometers of different resolution. J. Plant Physiol. 144, 307-313. doi: 10.1016/S01761617(11)81192-2

Wright, S. (1978). Evolution and the Genetics of Populations, Vol. 4. Variability within and among Natural Populations. Chicago: The University of Chicago Press, 580.

Xing, N., Fan, C., and Zhou, Y. (2014). Parental Selection of Hybrid Breeding Based On Maternal and Paternal Inheritance of Traits in Rapeseed (Brassica napus L.). PLoS ONE 9:e103165. doi: 10.1371/journal.pone.0103165

Conflict of Interest Statement: The authors declare that the research was conducted in the absence of any commercial or financial relationships that could be construed as a potential conflict of interest.

Copyright (c) 2015 Hatzig, Frisch, Breuer, Nesi, Ducournau, Wagner, Leckband, Abbadi and Snowdon. This is an open-access article distributed under the terms of the Creative Commons Attribution License (CC BY). The use, distribution or reproduction in other forums is permitted, provided the original author(s) or licensor are credited and that the original publication in this journal is cited, in accordance with accepted academic practice. No use, distribution or reproduction is permitted which does not comply with these terms. 